MS33-Prediction of molecular crystal structures

Chairs: Dr. Jacco van de Streek, Dr. Aurora Cruz-Cabeza
References:

[1] Sugden, I. et al. (2016). Acta Cryst. B72, 864-874.

[2] Kazantsev, A. V., et al. (2011). Process Systems Engineering, $1-42$.

Keywords: Crystal Structure Prediction, Molecular Modelling, Computational

\title{
MS33-P01
}

\section{New potentials for accurate and efficient $a b$ initio crystal structure prediction methods}

David Bowskill $^{1}$, Isaac Sugden ${ }^{1}$, Christina-Anna Gatsiou ${ }^{1}$, Claire S.

Adjiman $^{1}$, Constantinos C. Pantelides ${ }^{1}$

1. Department of Chemical Engineering, Imperial College London, London, United Kingdom

email: david.bowskill13@imperial.ac.uk

Many organic molecules of industrial interest exhibit crystalline polymorphism, forming multiple solid-state structures, each potentially displaying different physico-chemical properties. This makes the discovery of new polymorphs both desirable from an R\&D perspective and potentially disastrous from an operational standpoint. Consequently, understanding the crystal energy landscape of a commercial drug or chemical can be of great importance, especially if the compound is delivered in solid form, as is the case with suspended agrochemicals or oral pharmaceuticals. Current methods for crystal structure prediction (CSP) can be applied to molecules with limited conformational flexibility, although energy rankings are sometimes inaccurate. Furthermore, these methods become prohibitively expensive when the number of conformational degrees of freedom increases beyond 5 to 7.

We present recent progress on improving the accuracy and efficiency of a CSP method through changes to the interaction potential used, which enables the investigation of larger more flexible compounds. Our CSP method is based on the CrystalPredictor ${ }^{[1]}$ and CrystalOptimiser ${ }^{[2]}$ algorithms that a) efficiently explore the crystal energy landscape and b) accurately model leading contributions to lattice energy for predictions of stability and physical properties. We present a recently developed parameter estimation scheme for tailoring repulsive/dispersive contributions to the interaction potentials to crystal structure calculations, which leads to notable improvements in sublimation energy and structure reproduction with comparison to standard parameter sets.

We also introduce the concept of smoothed intramolecular potentials in CrystalPredictor, which offer near quantum-mechanical accuracy at much reduced computational cost. Such potentials make it possible to compute energy landscapes of increasingly large and conformationally flexible molecules through improved efficiency in the initial global search stage as well as increased accuracy in the resulting energy landscape. This, in turn, leads to a reduction in the number of structures that need to be further refined and hence further computational savings.

The above methods are demonstrated via application to both rigid and flexible molecules of industrial relevance. 